\title{
ALGEBRAIC GROUP-VARIETIES
}

\section{IACOPO BARSOTTI}

1. Definitions and preliminaries. Let $k$ be an algebraically closed field, and let $S$ be an $n$-dimensional projective space over $k$; the points of $S$ are the ordered sets $\left\{\xi_{0}, \cdots, \xi_{n}\right\}$ of elements of $k$, with the exception of the set $\{0, \cdots, 0\}$, and where two sets $\{\xi\},\left\{\xi^{\prime}\right\}$ are identified if $\xi_{i}^{\prime}=\rho_{\xi_{i}}$ for each $i$, and for some $\rho \in k$. An (algebraic) variety over $k$ is the set $V$ of all the points $\{\xi\}$ whose coordinates $\xi_{0}, \cdots, \xi_{n}$ satisfy a given finite set of homogeneous algebraic equations with coefficients in $k ; V$ is irreducible if it is not the join of two nonempty varieties, neither of which contains the other. The restriction to algebraically closed fields is not strictly necessary, but is very convenient for expository purposes, and will be kept in force throughout this address. The case in which $k$ is the complex field will be referred to as the classical case.

The (cartesian) product $V \times F$ of two varieties is defined in the usual way; it is a (pseudo-) variety embedded in a biprojective space, but it is also birationally equivalent (see below), in a one-to-one manner, to a variety (the Segre product) in the previous sense. A cycle on $V$ is an element of the free abelian group generated by the subvarieties of $V$; a cycle is effective if all its (irreducible) components appear in it with a positive coefficient (multiplicity). If $V, F$ are irreducible, an effective cycle $D$ on $V \times F$ is also called an algebraic correspondence between $F$ and $V$; to a point $P \in F$ corresponds the variety $D[P]$ consisting of all the $Q \in V$ such that $P \times Q \in D$; if $P$ is generic (that is, if it does not belong to a certain proper subvariety of $F$ ), and if all the components of $D$ have the same dimension and operate on the whole $F$, a certain multiplicity $e(U)$ can be attached to each component $U$ of $D[P]$; the cycle $\sum_{U} e(U) U$ on $V$ is then denoted by $D\{P\}$ (see [1] and [2] in the bibliography at the end of the paper).

The algebraic correspondence $D$ between $F$ and $V$ is called a rational mapping of $F$ into $V$ if $D\{P\}$ is a point, with multiplicity 1 , of $V$ for a generic $P \in F$; if $D$ is a rational mapping of $F$ into $V$ and also of $V$ into $F$, it is called a birational transformation, and $F, V$ are then said to be birationally equivalent $[31]$. These definitions, and this language, are used in $[1 ; 2 ; 3 ; 4 ; 5 ; 6 ; 7 ; 8 ; 31 ; 32]$; quite different,

An address delivered before the Vancouver meeting of the Society on June 18, 1955, by invitation of the Committee to Select Hour Speakers for Far Western Sectional Meetings; received by the editors June 25, 1955. 
but substantially equivalent, language is used by Weil and his school in $[12 ; 13 ; 18 ; 19 ; 20 ; 26 ; 29 ; 30]$.

The idea of algebraic correspondences is not limited to correspondences between varieties: it can be extended, with definite advantages, to algebraic correspondences between a field and a variety; this is done in $[1 ; 2]$, and systematically used in al lmy subsequent work.

In order to introduce the notion of group-variety, we shall first make the following convention: if $V$ is a variety, we shall denote by $V_{1}, V_{2}, \cdots$ a set of "copies" of $V$; if $x$ is any entity related to $V$, $x_{i}$ shall mean the copy of $x$ which is similarly related to $V_{i}$. Let then $V$ be an irreducible variety over $k$, and let $V_{1}, V_{2}, V_{3}$ be copies of $V$; a rational mapping of $V_{1} \times V_{2}$ into $V_{3}$ is called a law of composition on $V$; if $\{P, Q\}$ is a generic pair of points of $V$, and if $R_{3}=D\left[P_{1} \times Q_{2}\right]$, we shall write $R=P Q$; the law $D$ is called a normal law [30] if it is also a rational mapping of $V_{2} \times V_{3}$ into $V_{1}$, and of $V_{3} \times V_{1}$ into $V_{2}$, and if in addition it is associative, that is, if $(P Q) R=P(Q R)$ for any generic set $\{P, Q, R\}$ of points of $V$. We say that $V$ is a groupvariety with the law of composition $D$, if $D$ is a normal law, and if $V$ contains a proper subvariety $W$ such that $V-W$ (set-theoretical difference) is a group with the law of multiplication prescribed by $D$; $W$ is called the degeneration locus of $V$. It is easily verified that the group $V-W$ can be interpreted, in more than one way, as a group of birational transformations of $V$, or of automorphisms of $k(V)$ ( = field of the rational functions on $V$ ) over $k$. When this is done, in a given way, $\sigma_{P}$ shall denote the automorphism related to $P \in V-W$; it satisfies the relation $\sigma_{P} Q=P Q$ for $Q \in V-W$.

I shall not insist on the obvious definitions of direct product $V \times F$ of two group-varieties, of group-subvariety, and of invariant groupsubvariety; it must be noted, however, that a group-subvariety of a group-variety need not be irreducible. An immediate example of group-variety is given by an $n^{2}$-dimensional projective space $G$ : if the nonhomogeneous coordinates of the points of $G$ are arrayed in a matrix of order $n$, the matrix-product establishes a law of composition on $G$, which turns $G$ into a group-variety whose degeneration locus consists of the hyperplane at infinity, and of the subvariety of the points represented by matrices with vanishing determinant. This group-variety $G$, and its irreducible group-subvarieties, form an important class of group-varieties, which I have called Vessiot varieties, for the role they play in the algebraic Picard-Vessiot theory of differential equations $[16 ; 17]$. Vessiot varieties are usually noncommutative, but the following special commutative cases are noteworthy: 
(1) vector varieties; these are isomorphic (that is, birationally equivalent as varieties, and isomorphic as groups) to direct products of finitely many straight lines, the law of composition on each straight line being given by the addition of the abscissae; (2) logarithmic varieties; these are isomorphic to direct products of finitely many straight lines, the law of composition on each straight line being given by the multiplication of the abscissae; (3) periodic varieties; these are commutative group-varieties $G$ having the property that $P^{b}=E$ (identity) for each nondegenerate $P \in G$, and for a suitable positive integer $e$, independent of $P$; it is proved in [5] that periodic varieties exist only over fields of characteristic $p>0$, in which case the smallest possible $e$ is a power of $p$; the vector varieties over a field of characteristic $p>0$ are all and only the periodic varieties of period $p$ [5]. It is rather easy to prove that vector varieties and logarithmic varieties are Vessiot varieties; in $[16 ; 17]$ it is also proved that any commutative Vessiot variety is the direct product of a logarithmic variety and a vector, or a periodic, variety, depending on whether $k$ has zero or positive characteristic. The proof of the fact that every periodic variety is a Vessiot variety is given in [6]; an announcement of the same fact is contained in [26].

Vector varieties, logarithmic varieties, and periodic varieties share the property of being rational, that is, birationally equivalent to a projective space; any Vessiot variety over a field of characteristic zero has the same property (result unpublished) $;^{1}$ most probably, the same is true for any characteristic, but no proof of this fact is available yet.

All Vessiot varieties have a nonempty degeneration locus; the last, and most important, example of group-varieties is given by the abelian varieties, which are, by definition, the group-varieties with empty degeneration locus; they are necessarily commutative [30].

2. Historical note and statement of the problems. The study of problems related to, or stemming from properties of group-varieties, mainly in the classical case, has formed, and still forms, the historical center at which several branches of mathematics meet. Abelian varieties originate in the celebrated problem of the inversion of what we now call the abelian integrals of the first kind on an algebraic curve of genus $g \geqq 1$; it now appears [27] that other types of commutative group-varieties originate in the problem of the inversion of integrals

${ }^{1}$ Added February, 1956. This result was published at about the same time this address was delivered; see C. Chevalley, On algebraic group-varieties, J. Math. Soc. Japan vol. 6 (1954) p. 303. 
of the second and third kind (see $\S \S 3$ and 4 ). For $g=1$, the solution of the first problem is expressed in the theory of elliptic functions; for $g>1$, the beautiful theory of abelian functions (or meromorphic functions of $g$ complex variables with $2 g$ periods) gives a transcendental tool of primary importance. The deeper results in this theory were obtained by transcendental and topological means, of which Conforto has given, in [14], a modern and rigorous account. The translation and generalization of the theory of abelian varieties to the abstract case (arbitrary $k$ ) has been made possible by the pioneering work of Zariski $[31 ; 32]$, and by the revolutionary new tools which he forged in the arithmetical method in algebraic geometry; the actual reconstruction, within this framework, of the theory of abelian varieties, has been beautifully accomplished by Weil in a true masterpiece of modern mathematics [30].

Already in the classical works a basic problem manifests itself, although at times it is blurred by the general lack of rigor: it is not difficult to prove that, by means of abelian functions, an analytic and generally one-to-one correspondence can be established between a toroid (or the parallelotope of the periods) and an algebraic variety $V$; this, of course, establishes a normal law on $V$, reflecting the addition of the arguments of the abelian functions. The problem is to find whether $V$ can be selected in such a way that the correspondence is strictly one-to-one, in which case $V$ will be a group-variety. No rigorous solution to this problem can be found in the classical literature, although [14] contains a clear statement of it, and some useful hints concerning its solution. When the theory of group-varieties is put on a purely algebraic basis, the necessity of finding a solution to the analogous problem becomes even more acute, so acute in fact that Weil has developed, in [29], a new tool (the "abstract varieties") which allows him to by-pass the problem itself, at the cost of abandoning the geometrical interpretation of varieties as subsets of a projective space. Shortly after, however, the problem was completely solved in [4]; actually, when only abelian varieties are concerned, the solution is surprisingly simple, and a particular case of it is also given in $[13] .{ }^{2}$

Before closing the subject of abelian varieties, it should be noted that through the theory of Riemann matrices in the classical case, or the ring of endomorphisms of an abelian variety in the abstract case, the theory of abelian varieties provides a powerful tool for the solution of difficult questions of analytic number theory.

2 A proof for the case of abelian varieties appears also in: [20]. In the classical case, and for abelian varieties only, the result is also contained in: K. Kodaira, On Kähler varieties of restricted type, Ann. of Math. vol. 60 (1954) p. 28. 
Both abelian varieties and Vessiot varieties fit naturally (in the classical case) in the theory of Lie groups, but Vessiot varieties seem to have received more attention by the specialists on Lie groups; of course, the much discussed "classical groups" are, or are related to, very particular cases of Vessiot varieties. However, not much attention seems to have been paid to those groups of matrices which are algebraic, and to their algebraic nature, in spite of the fact that the theory of invariants is an algebraic theory. The only recent and rigorous works on the subject seem to be [10], [16] and [17]; while the precise scope and purpose of [10] is still not too clear to me, [16] and [17] contain abundant results on Vessiot varieties, as well as on the more immediate purpose of their author, namely the algebraic and rigorous reconstruction of the Picard-Vessiot theory of differential equations.

The main problem on Vessiot varieties is their classification and construction; in the classical case, both are achieved rather neatly by shifting the emphasis from the varieties to their Lie algebras; this artifice does not work in characteristic $p>0$, and [10] offers convincing proof of this statement. The reason for this failure is to be sought, in my opinion, in the fact that the coefficients of the Taylor expansion of a function over a field of positive characteristic are not obtainable by iterated derivations. It is also my opinion that a method dealing with these coefficients directly, rather than with the set of the derivations, would completely reëstablish the analogy with the classical case; ; a modest example of this is given in $\S 6$ of [5].

We now come to the main problem on general group-varieties, namely their classification and construction (or structure). As usual in mathematics, this problem is to be considered solved when its solution is made to depend on problems which, in the logical or historical development of the theory, are parts of a "previous theory". Thus, abelian varieties and Vessiot varieties must be considered as "known" when investigating the structure of general group-varieties. It is quite natural to investigate first the structure of commutative group-varieties, and later the structure of general group-varieties; I shall say immediately that the second, more general problem has been completely neglected until now, except in so far as noncommutative group-varieties fit into the general theory of Lie groups. The first, more restricted problem, has fared a little better, since commutative group-varieties lend themselves, in the classical case, to be studied by means of quasi-abelian functions, that is, meromorphic

${ }^{3}$ This program has been realized, for "formal Lie groups", in: J. Dieudonné, Groupes de Lie et hyperalgèbres de Lie sur un corps de caractéristique $p>0$, Comment. Math. Helv. vol. 28 (1954) p. 87. 
functions of $g$ complex variables, which satisfy an addition theorem in the sense of Weierstrass (and which consequently have $\mu \leqq 2 g$ periods). An extensive literature on this subject is to be found in [27]. [27] and [28] are the most recent contributions to the subject along classical lines, and apparently the only classical contributions with a distinctly geometrical outlook (although the author skilfully avails himself of transcendental tools). It is unfortunate that [27] partakes of those shortcomings which for a few decades stifled serious progress in algebraic geometry, namely the lack of clear definitions and of rigorous proofs; for instance, it seems to me that group-varieties are never even taken into consideration in [27], the subject always being varieties with a commutative normal law.

The general method followed by Severi in [27] can certainly be made logically rigorous, translated into algebraic language, and generalized to arbitrary characteristic. This translation and generalization has been undertaken in $[24 ; 25 ; 26]$, and it is my understanding that it has now born the expected fruit.

3. Preparatory and structural results. I will now briefly present the main results of my studies on the subject in the course of the last three years. First of all, the free interchange of "varieties with a normal law of composition" and "group-varieties" is made possible by the following result $[4]$ :

(1) Theorem. Let $V$ be an (absolutely) irreducible variety over the (not necessarily algebraically closed) field $k$, and let $D$ be a normal law of composition on $V$. There exists a variety $G$ over $k$, birationally equivalent to $V$, such that $G$, with the law of composition induced by $D$, is a group-variety. $G$ is unique but for isomorphisms.

The most immediate application of (1) is to the homomorphism theorems $[5 ; 12]$; a homomorphism $\alpha$ of a group-variety $G$ into a group-variety $G^{\prime}$ is a rational mapping of $G$ into $G^{\prime}$ which is also, as far as nondegenerate points are concerned, a group-homomorphism; the kernel of $\alpha$ is the smallest subvariety of $G$ which contains all the nondegenerate points of $G$ which map on the identity of $G^{\prime}$. The homomorphism $\alpha$ is separable if $\alpha\{P\}$ has no component of multiplicity $>1$ for a generic $P \in G^{\prime}$. I will not state here the three homomorphism theorems, which are analogous to those of group-theory; the only difference lies in the fact that there may exist homomorphisms (purely inseparable) whose kernel is the identity, but which nonetheless are not isomorphisms. This is not the case, however, if the characteristic of $k$ is zero. 
All the structural theorems for commutative group-varieties depend on two preparatory results [5], the first of which reads:

(2) Lemma. A nonabelian commutative group-variety of dimension $>1$ has some positive dimensional proper group-subvariety.

The proof of this result is approximately as follows: if $G$ is the given variety, and $F$ its nonempty degeneration locus, and if $\operatorname{dim} G=n>1$, let $X$ be an $(n-1)$-dimensional irreducible subvariety of $G$. If there exist infinitely many $P \in G-F$ such that $\sigma_{P}$ leaves $X$ invariant, they lie on the desired group-subvariety. If not, $\sigma_{P} X$ depends on $P$, and when $P$ approaches a suitable point of $F, \sigma_{P} X$ approaches a cycle $Y$ which does have the previous property. Of course, "approaching" is replaced by a suitable algebraic notion.

Before stating the second preparatory result, we must introduce, in analogy to group-theory, the concept of factor set. Let $G, V$ be commutative group-varieties over $k$, and let $G_{1}, G_{2}$ be copies of $G$. Let $\gamma$ be a rational mapping of $G_{1} \times G_{2}$ into $V$, such that the relations $\left(\gamma\left[P_{1} \times Q_{2} R_{2}\right]\right)\left(\gamma\left[Q_{1} \times R_{2}\right]\right)=\left(\gamma\left[P_{1} Q_{1} \times R_{2}\right]\right)\left(\gamma\left[P_{1} \times Q_{2}\right]\right)$ and $\gamma\left[P_{1} \times Q_{2}\right]$ $=\gamma\left[Q_{1} \times P_{2}\right]$ are satisfied for a generic set $\{P, Q, R\}$ of points of $G$; assume, moreover, $\gamma\left[E_{1} \times E_{2}\right]$ to be a nondegenerate point of $V$, if $E$ is the identity of $G$. Then $\gamma$ is called a factor set of $G$ into $V$. The set of all such $\gamma$ 's is denoted by $\Gamma(G, V)$, and becomes a commutative group when a "natural" law of multiplication is introduced. The factor set $\gamma$ is associate to the identity, or $\gamma \sim 1$, if there exists a rational mapping $\mu$ of $G$ into $V$ such that $\gamma\left[P_{1} \times Q_{2}\right]=(\mu[P])(\mu[Q])(\mu[P Q])^{-1}$ for generic $P, Q \in G$. The set $\Gamma_{0}(G, V)$ of the $\gamma \sim 1$ is a subgroup of $\Gamma(G, V)$. If $\gamma \in \Gamma(G, V)$, we can establish a normal law of composition $D$ on the cartesian product $G \times V$ by setting $(P \times Q)(R \times S)$ $=P R \times Q S \gamma\left[P_{1} \times R_{2}\right]$, where $P, R \in G$, and $Q, S \in V$. The groupvariety determined by $G \times V$ and $D$ according to (1) is called a crossed product of $G$ and $V$, and denoted by $\{G, V, \gamma\}$; it is isomorphic to the direct product $G \times V$ if and only if $\gamma \in \Gamma_{0}(G, V)$. Now, the result which we have in mind (and whose proof rests essentially on Lüroth's theorem) is the following [5]:

(3) Lemma. Let $G$ be a commutative group-variety over $k$, and let $V$ be a rational 1-dimensional irreducible group-subvariety of $G$ (that is, a straight line, either additive or multiplicative); then $G$ is a crossed product of $G / V$ and $V$.

Here, $G / V$ is the image of $G$ in the homomorphism whose kernel is $V$. From (2), (3), and from a result of [30], stating that any rational mapping of a rational variety $R$ into an abelian variety maps the 
whole $R$ onto a point, the complete structural theorems for commutative group-varieties follow, namely [5]:

(4) ThEOREM. A commutative group-variety Gover $k$ contains a unique maximal irreducible rational group-subvariety $V ; A=G / V$ is abelian, and $G$ is a crossed product of $A$ and $V$.

(5) Theorem. A commutative rational group-variety $G$ over $k$ contains a unique maximal irreducible logarithmic subvariety $L$, and a unique maximal irreducible vector (if $k$ has characteristic zero) or periodic (if $k$ has positive characteristic) subvariety $V$; moreover, $G$ is isomorphic to the direct product $L \times V$.

(6) Theorem. $A$ periodic group-variety of dimension $n+1$ over $k$ is the crossed product of a periodic group-variety of dimension $n$, and of a 1-dimensional vector variety.

The case of noncommutative group-varieties lends itself to an attack from two directions, which combine to give a rather strong result, although not as strong as (4). The first of these (described in [5]) is brought about by considering two isomorphic general points $X, Y$ of a group-variety $G$ over $k$ (so that $X, Y$ will have coordinates in a suitable extension of $k$ ), and by expanding the coordinates of $X^{-1} Y X$ in series of powers of the coordinates of $Y$. The coefficients of these power series are rational functions of the coordinates of $X$, and can be taken as coordinates of a general point of a new variety $V$. It turns out that if the series are truncated at a suitable point, $V$ is a Vessiot variety, image of $G$ in a homomorphism whose kernel is the center $C$ of $G$. If the characteristic of $k$ is zero, two concomitant facts concur to make the picture particularly simple, namely: (1) the series can be truncated after the first powers, and (2) $V$ is necessarily isomorphic to $G / C$, and can accordingly be called the adjoint variety. Both properties are, substantially, consequences of the applicability of the theory of Lie algebras. If the characteristic of $k$ is positive, neither property is necessarily true, and examples of their failure are readily constructed. In this case, $V$ is called the stem of $G$; it remains true, however, that $G / C$ is a Vessiot variety, but this is a consequence of the second method of attack [6].

The other line of attack (described in [6]) involves the consideration of the set of the elements $x$ of $k(G)$ (rational functions on $G$ ) which have no poles on $G$ outside the degeneration locus; such elements form a ring, whose field of quotients is also the field of rational functions on a Vessiot variety $F$, homomorphic image of $G$ in a separable homomorphism $\alpha$. In the language of the theory of group representations, $\alpha$ is a maximal representation of $G$. Various conse- 
quences can be drawn from these two results, of which the most important are [6]:

(7) Theorem. ${ }^{4} A$ group-variety $G$ over $k$ contains a unique maximal Vessiot subvariety $H ; H$ is invariant in $G$, and $G / H$ is abelian.

(8) CoRollary. Every rational group-variety is a Vessiot variety.

Result (7) is not as strong as the analogous result for commutative group-varieties, namely (4). If (8) could be inverted, it would be possible to refine (7) in a manner similar to (4); in any case, the following generalization of two results of [30] is helpful [5]:

(9) Theorem. Let $A$ be an abelian group-subvariety of a groupvariety $G$; then $A$ is a subvariety of the center of $G$, and $G$ contains an invariant irreducible group-subvariety $B$ such that the intersection $A \cap B$ is a finite set of points.

4. Factor sets. Result (4) gives complete information on all the possible $G$ if the structure of $\Gamma(A, V) / \Gamma_{0}(A, V)$ is known. The study of this group forms the object of $\$ 4$ of [5], of [7], and, in part, of [8]. It can be easily shown that it is sufficient to study the structure of $\Gamma(A, V) / \Gamma_{0}(A, V)$ for the simple case in which $V$ is 1-dimensional, and either logarithmic or a vector variety.

In the classical case, let us denote by $D_{,} D_{e}, D_{1}, D_{2}$ the additive groups of, respectively, the closed differentials on $A$ with integral residues, ${ }^{5}$ the exact differentials on $A$, the closed differentials of the first kind on $A$, and the closed differentials of the second kind on $A$; let $D_{l}$ denote the additive group of the differentials of the type $d x / x$, for $0 \neq x \in k(A)$. Then it can be proved by transcendental means (and is implicitly stated in [27]) that if $V$ is a 1-dimensional vector variety, $\Gamma(A, V) / \Gamma_{0}(A, V)$ is isomorphic to $D_{2} / D_{1}+D_{e}$, while $\Gamma(A, V) / \Gamma_{0}(A, V)$ is isomorphic to $D / D_{2}+D_{l}$ if $V$ is a 1 -dimensional logarithmic variety. In the first case, it is also known, by transcendental and topological means, that $D_{2} / D_{1}+D_{e}$ is a $k$-module of order $n=\operatorname{dim} A$, so that the same is true of $\Gamma(A, V) / \Gamma_{0}(A, V)$. Now, this has been proved in [7], by algebraic means only, for any $k$ of characteristic zero, and leads to the following result:

(10) TheORem. Let $A$ be an n-dimensional abelian variety over the field $k$ of characteristic zero; let $V$ be a 1-dimensional vector variety over $k$.

4 Added May, 1956. I have been informed that a proof of this result, valid for the case of characteristic zero, was announced by C. Chevalley in the summer of 1953 . [5].

6 This condition concerning the residues was erroneously left unmentioned in $\$ 7$ of 
Then $\Gamma(A, V) / \Gamma_{0}(A, V)$ is a k-module of dimension $n$, isomorphic to $D_{2} / D_{1}+D_{e}$.

The core of the algebraic proof of (10) lies in the fact that $D_{1}$ is the set of all the invariant differentials on $A$ (invariant under the transformations $\sigma_{P}$ for $P \in A$ ), while $\mathscr{D}_{2}$ is the set of all the semi-invariant closed differentials on $A$, namely of those closed differentials $\omega$ such that $\sigma_{P} \omega-\omega \in D_{e}$ for any $P \in A$.

The second case, or the case in which $V$ is logarithmic, could probably also be treated by means of the differentials; but there is a more natural and geometrical manner, which I shall explain after introducing a few new concepts.

Quite in general, let $V$ be a normal [31] $n$-dimensional irreducible variety over $k$, and let $Z(V)$ be the group of the $(n-1)$-dimensional cycles on $V$. An element $z \in Z(V)$ is algebraically equivalent to zero, or $z \approx 0$, if there exist an irreducible variety $F$ over $k$, an algebraic correspondence $D$ between $F$ and $V$, and two points $P, Q$ of $F$, such that $z=D\{P\}-D\{Q\}$; if $F$ can be selected to be rational, and $D$ can be selected so that it operates on the whole $F, z$ is said to be linearly equivalent to zero, in symbols $z \sim 0$. Finally, $z$ is arithmetically equivalent to zero, or $z \equiv 0$, if the intersection $[3](z \cap C, V)$ of $z$ with any curve $C$ of $V$ has order zero whenever it exists. The sets of, respectively, the cycles $z \sim 0, z \approx 0, z \equiv 0$ will be denoted by $\mathfrak{L}(V), \mathfrak{e}(V), a(V)$ respectively; they are subgroups of $\mathcal{Z}(V)$, and satisfy the inclusion $\mathscr{L}(V)$ $\subseteq \mathfrak{e}(V) \subseteq \mathfrak{Q}(V) \subset \mathcal{Z}(V)$. The following relations have been proved within the framework of classical algebraic geometry, and by the combined efforts of, mainly, Picard, Castelnuovo, Poincaré, Enriques, Severi and Lefschetz: (a) $Z(V) / Q(V)$ is a finite free module over the ring of integers; (b) $a(V) / \mathfrak{e}(V)$ is a finite group, whose order is the torsion of $V ;$ (c) $\mathfrak{e}(V) / \mathfrak{L}(V)$ is isomorphic, as a group, and "birationally" so, in a well determined way, to an abelian variety, the Picard variety of $V$. A mixture of properties (a) and (b), namely the fact that $Z(V) / \mathcal{C}(V)$ is a finite module over the ring of integers, has been rigorously reëstablished, by algebraic means and for any characteristic, by Néron in [22]; property (c) has also been reëstablished under the same conditions in [18] and [19] by Matsusaka, or in [23] (also in an unpublished work by the author). In the case in which $V=A=$ an abelian variety, property (a) can be proved independently [8]; property (b) follows from the fact that (by (19) of [8]) every element of $\mathfrak{Q}(A) / \mathfrak{e}(A)$ has a finite, bounded, period, and that consequently (by Proposition 4 of [22]) is itself finite. As a matter of fact, the quoted results imply that $\mathbb{Q}(A)=\mathfrak{C}(A)$ if $k$ has characteristic zero, and that $\mathfrak{Q}(A) / \mathfrak{e}(A)$ has an order which is a power of the 
characteristic $p$ of $k$ if $p>0$. Finally, as far as property (c) is concerned, it is proved in [8] that the Picard variety of $A$ is isogenous to $A$, namely, has the same dimension of $A$ and is a homomorphic image of $A$. It must be noted also that if $z \in \mathcal{Z}(A)$, then $z \equiv 0$ if and only if $z$ is linearly equivalent to each $\sigma_{P z}$ for $P \in A$; this definition of the equivalence $\equiv$ is profitably used in [30], and its identity with arithmetic equivalence is proved in [8].6

This being established, we have $[5 ; 8]$ :

(11) THEOREM. Let $A$ be an abelian variety over the field $k$ of characteristic $p$; let $V$ be a 1-dimensional logarithmic variety over $k$. Then $\Gamma(A, V) / \Gamma_{0}(A, V)$ is isomorphic to $\mathbb{Q}(A) / \mathfrak{L}(A)$; this, in turn, is isomorphic to the group of the points of the Picard variety $B$ of $A$ if $p=0$. If $p>0, a(A) / \mathscr{L}(A)$ contains a subgroup isomorphic to $B$, and having in $Q(A) / \mathfrak{L}(A)$ an index which is a (finite) power of $p$.

The only remaining case is that in which $k$ has positive characteristic, and $V$ is a vector variety. Work on this case is not completed, but what there is would indicate that a result similar to (10) holds true. The method of proof, however, is completely different, and is based on the consideration of the $k$-module $D_{1} \cap D_{e}$, which in this case is not necessarily zero-dimensional; the same method, through the consideration of the group $D_{1} \cap D_{l}$, throws light on the value of the torsion of $A$, or of the index mentioned in (11). ${ }^{7}$

\section{BIBLIOGRAPHY}

1. I. Barsotti, Algebraic correspondences between algebraic varieties, Ann. of Math. vol. 52 (1950) p. 427; see also Errata, ibid. vol. 53 (1951) p. 587.

2. - Local properties of algebraic correspondences, Trans. Amer. Math. Soc. vol. 71 (1951) p. 349.

3. - Intersection theory for cycles of an algebraic variety, Pacific Journal of Mathematics vol. 2 (1952) p. 473. 236.

4. - A note on abelian varieties, Rend. Circ. Mat. Palermo vol. 2 (1953) p.

5. - Structure theorems for group-varieties, Annali di Matematica Pura ed Applicata vol. 38 (1955) p. 77.

6. - Un teorema di struttura per le varietd gruppali, Rend. Acc. Naz. Lincei vol. 18 (1955) p. 43.

7. - Factor sets and differentials on abelian varieties, to appear.

8. - Il teorema di dualitd per le varietd abeliane ed altri risultati, Rendiconti di Matematica e delle sue Applicazioni vol. 13 (1954) p. 98.

${ }^{6}$ See (16) of [8]; the result of $n .72$ of [30] mentioned in the proof of (16) of [8] is valid only when $A$ is simple. A standard manipulation shows, however, that if (16) of [8] is valid when $A$ is simple, it is also valid for any $A$.

7 Added February, 1956. It has now been proved that result (10), with the exception of its last three words, remains true when $k$ has characteristic $\neq 0$, and that the index mentioned in (11) is equal to 1 . 
9. C. Chevalley, Theory of Lie groups, Princeton, 1946.

10. - Theorie des groupes de Lie, II, Groupes algébriques, Paris, 1951.

11. - Introduction to the theory of algebraic functions of one variable, New York, 1951.

12. W. L. Chow, On the quotient variety of an abelian variety, Proc. Nat. Acad. Sci. U.S.A. vol. 38 (1952) p. 1039.

13. — , The jacobian variety of an algebraic curve, Amer. J. Math. vol. 76 (1954) p. 453. 1942.

14. F. Conforto, Funzioni abeliane e matrici di Riemann, parte prima, Roma,

15. - Problèmes résolus et non résolus de la thêorie des fonctions abéliennes dans ses rapports avec la géométrie algébrique, Coll. de Géom. Alg., Liège, 1952.

16. E. R. Kolchin, Algebraic matric groups and the Picard-Vessiot theory of homogeneous linear ordinary differential equations, Ann. of Math. vol. 49 (1948) p. 1.

17. - On certain concepts in the theory of algebraic matric groups; Ann. of Math. vol. 49 (1948) p. 774.

18. T. Matsusaka, On the algebraic construction of the Picard variety, Jap. J. Math. vol. 21 (1951) p. 217.

19. - On the algebraic construction of the Picard variety (II), Jap. J. Math. vol. 22 (1952) p. 51.

20. - Some theorems on abelian varieties, Natural Science Reports of the Ochanomizu University vol. 4 (1953) p. 22; see also A remark . . ., ibid., p. 172.

21. H. Morikawa, Cycles and endomorphisms of abelian varieties, Nagoya Mathematical Journal vol. 7 (1954) p. 95.

22. A. Néron, Problèmes arithmétiques et géométriques rattaches a la notion de rang d'une courbe algébrique dans un corps, Bull. Soc. Math. France vol. 80 (1952) p. 101.

23. A. Néron and $\mathrm{P}$. Samuel, La variété de Picard d'une variété normale, Annales de l'Institut Fourier vol. 4 (1952) p. 1.

24. M. Rosenlicht, Equivalence relations on algebraic curves, Ann. of Math. vol. 56 (1952) p. 169.

25. - Differentials of the second kind for algebraic function fields of one variable, Ann. of Math. vol. 57 (1953) p. 517.

26. - Generalized jacobian varieties, Ann. of Math. vol. 59 (1954) p. 505.

27. F. Severi, Funzioni quasi abeliane, Pont. Acad. Sci. Scripta Varia, vol. 4, 1947.

28. - Sulla caratterizzazione dei corpi di funzioni quasi abeliane, Convegno Intern. Geom. Diff., 1953, p. 21.

29. A. Weil, Foundations of algebraic geometry, Amer. Math. Soc. Colloquium Publications, vol. 29, 1946.

30. - Variêtés abéliennes et courbes algêbriques, Paris, 1948.

31. O. Zariski, Foundations of a general theory of birational correspondences, Trans. Amer. Math. Soc. vol. 53 (1943) p. 490.

32. - The concept of simple point of an abstract algebraic variety, Trans. Amer. Math. Soc. vol. 62 (1947) p. 1.

University of Pittsburgh and University OF SOUThERN CALIFORNIA 